\title{
A novel small diameter nanotextile arterial graft is associated with surgical feasibility and safety and increased transmural endothelial ingrowth in pig
}

John Joseph ${ }^{1,2}$, Vito Domenico Bruno ${ }^{1}$, Nadiah Sulaiman ${ }^{1}$, Alexander Ward ${ }^{1}$, Thomas W. Johnson?', Helna Mary Baby², Praveen Kerala Varma ${ }^{3}$, Rajesh Jose ${ }^{3}$, Shantikumar V. Nair ${ }^{2}$, Deepthy Menon²*, Sarah Jane George ${ }^{1 *}$ and Raimondo Ascione ${ }^{1 *}$ (D)

\begin{abstract}
Globally, millions of patients are affected by myocardial infarction or lower limb gangrene/amputation due to atherosclerosis. Available surgical treatment based on vein and synthetic grafts provides sub-optimal benefits. We engineered a highly flexible and mechanically robust nanotextile-based vascular graft (NanoGraft) by interweaving nanofibrous threads of poly-L-lactic acid to address the unmet need. The NanoGrafts were rendered impervious with selective fibrin deposition in the micropores by pre-clotting. The pre-clotted NanoGrafts ( $4 \mathrm{~mm}$ diameter) and ePTFE were implanted in a porcine carotid artery replacement model. The fibrin-laden porous milieu facilitated rapid endothelization by the transmural angiogenesis in the NanoGraft. In-vivo patency of NanoGrafts was 100\% at 2- and 4-weeks, with no changes over time in lumen size, flow velocities, and minimal foreign-body inflammatory reaction. However, the patency of ePTFE at 2-week was $66 \%$ and showed marked infiltration, neointimal thickening, and poor host tissue integration. The study demonstrates the in-vivo feasibility and safety of a thin-layered vascular prosthesis, viz., NanoGraft, and its potential superiority over the commercial ePTFE.
\end{abstract}

Keywords: Nanotextile, Small diameter vascular grafts, Endothelialisation, Nanofibers, Electrospinning, In-vivo feasibility, Coronary surgery, Vascular surgery, Nanotextile vascular prosthesis, Vascular graft failure, Tissue engineering

*Correspondence: deepthymenon@aims.amrita.edu; S.J.George@bristol.

ac.uk; R.Ascione@bristol.ac.uk

${ }^{1}$ Bristol Heart Institute and Translational Biomedical Research Centre,

Faculty of Health Science, University of Bristol, Bristol BS2 8HW, UK

${ }^{2}$ Centre for Nanosciences \& Molecular Medicine, Amrita Vishwa

Vidyapeetham, Kochi 682 041, India

Full list of author information is available at the end of the article give appropriate credit to the original author(s) and the source, provide a link to the Creative Commons licence, and indicate if changes were made. The images or other third party material in this article are included in the article's Creative Commons licence, unless indicated otherwise in a credit line to the material. If material is not included in the article's Creative Commons licence and your intended use is not permitted by statutory regulation or exceeds the permitted use, you will need to obtain permission directly from the copyright holder. To view a copy of this licence, visit http://creativecommons.org/licenses/by/4.0/. The Creative Commons Public Domain Dedication waiver (http://creativecommons.org/publicdomain/zero/1.0/) applies to the data made available in this article, unless otherwise stated in a credit line to the data. 


\section{Graphical Abstract}

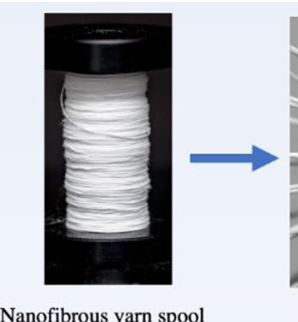

Nanofibrous yarn spool

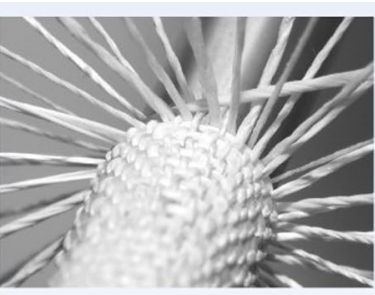

Circular weaving of nanofibrous yarns

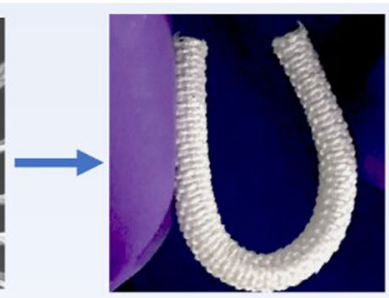

Highly flexible tubular Nanograft

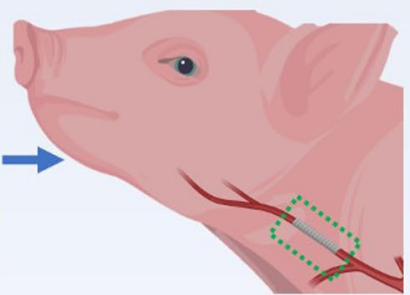

NanoGraft implanted in porcine carotid artery

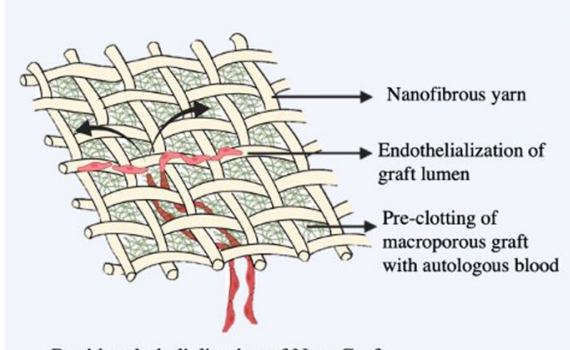

Rapid endothelialization of NanoGraft by transluminal capillary ingrowth

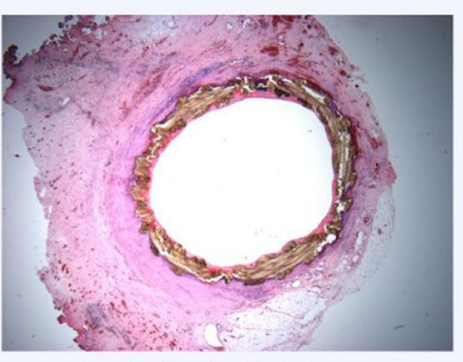

Thrombus free NanoGraft after 2 weeks implantation

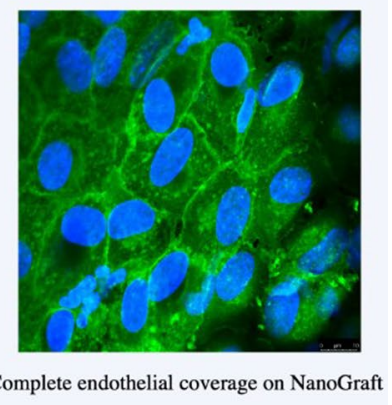

\section{Introduction}

Worldwide $>220$ million patients are at risk of stroke, myocardial infarction, lower limb gangrene, and amputation due to severe atherosclerosis, a disease mainly affecting arteries with $<6 \mathrm{~mm}$ diameter [1]. The established surgical treatments for the most severe forms of coronary and peripheral artery disease include coronary artery bypass grafting (CABG) and lower limb peripheral artery bypass grafting (PABG) using autologous or synthetic small diameter grafts [1-3]. About $80 \%$ of the small diameter grafts consist of either autologous saphenous vein grafts (SVGs) or synthetic expanded polytetrafluoroethylene grafts (ePTFE) [4]. However, these grafts encounter $10-15 \%$ early thrombosis within 1 month, and $50 \%$ occlusion at 1-year due to intimal hyperplasia for CABG-SVGs [4-8] and show similar longevity following PABG for both SVGs and PTFE grafts [9-11]. Hence, there is a critical clinical need for more effective small diameter arterial conduits. Available synthetic ePTFE and polyethylene terephthalate (PET) grafts made from non-degradable materials feature lower patency rates of $<50 \%$ at 1 -year due to poor endothelialisation, marked intimal thickening, and acute thrombosis [12]. This is triggered by the low flow through the small cross-sectional area and high turbulence at the anastomotic sites. All of these can be attributed mainly to the mismatch in mechanical properties with the native vessel $[13,14]$. Different strategies of engineering small vascular grafts have utilized biodegradable polymers, surface modification techniques, tissue engineering, and decellularization of xenografts $[15,16]$. None of these approaches have proven success. They fail to address the essential goals for longevity, namely, (i) mechanical properties analogous to the native vessel and (ii) endothelialisation of the internal wall surfaces of the graft to maintain homeostasis and mitigate intimal hyperplasia.

To address these limitations, our group at Amrita (India) developed a novel concept of biodegradable, flexible vascular graft using bundles of nanofibrous threads called yarns [17], which comprises of thousands of individual nanofibers woven together to form nanotextile conduits of predefined small diameter [18]. Woven nanotextiles comprises of interwoven longitudinal and transverse nano-yarns, which collectively enhance the surface area and mechanical property of the vascular graft. Non-woven fibers do not provide the requisite mechanical strength and integrity to serve as a vascular conduit. The use of polymeric nano-yarns offered scalable manufacturing and precise modulation of graft porosity in the micron-scale, which are vital attributes of this patented technology [18]. In this work, this novel vascular graft (NanoGraft) was subjected to preliminary preclinical validation of safety and efficacy at Bristol (UK) under physiological arterial conditions involving in-vitro and in-vivo feasibility testing in a large-animal porcine model of carotid artery replacement. In addition, mechanisms of in-vivo engraftment were studied. 


\section{Materials and methods}

The full version of Materials and Methods is shown in the Additional file 1.

\section{Manufacturing of tubular nanotextiles}

Polymeric yarns were produced from poly(L-lactic acid) (PLLA) previously subjected to electrospinning. These were then utilized to make the NanoGraft using a weaving system designed and fabricated in-house [17, 18]. The NanoGraft consisted of a single circumferential yarn that interweaves through multiple longitudinal yarns. The total number of longitudinal yarns used for developing a tightly woven nanotextile was calculated based on the predefined diameter of the conduit. To obtain a $4 \mathrm{~mm}$ vascular conduit, a total of 47 longitudinal yarns were interwoven by a single circumferential yarn to attain a tightly woven graft with 330 interweaves per unit area $\left(\mathrm{cm}^{2}\right)$.

\section{NanoGraft pre-clotting protocol}

As the woven NanoGraft was intended for arterial applications, it was subjected to a pre-clotting protocol as described by Sauvage et al. [19]. to prevent leakage. Briefly, the graft was soaked in $10 \mathrm{ml}$ of fresh autologous pig blood and incubated at $37{ }^{\circ} \mathrm{C}$ for the next $10 \mathrm{~min}$ to allow transmural fibrin formation. Next, it was flushed twice with $10 \mathrm{ml}$ of autologous blood to remove any lumi$\mathrm{nal} /$ mural debris and incubated for $30 \mathrm{~s}$. Finally, the graft was pressurized at $120 \mathrm{mmHg}$ to mimic arterial pressure using heparinized porcine autologous blood (10 $\mathrm{ml}$ blood containing 4000 IU Heparin). In this final step, Heparin was used to neutralize any unreacted thrombin.

\section{Dynamic bench testing of physical properties}

The physical properties of the nanotextile conduit were evaluated at the Bristol Heart Institute under arterial pulsatile flow conditions using a bioreactor (TGT DynaGen ${ }^{\circledR}$ Series, USA) primed with fresh heparinized porcine blood (Additional file 1: Fig. S2, Additional file 2: Video 1). To ensure safety, the dynamic testing was conducted at physiological conditions (blood pressure of 120/80 $\mathrm{mmHg}, \sim 72$ cycles/min) and accelerated conditions (blood pressure of 400/350 $\mathrm{mmHg}, \sim 350$ cycles/ min) to test mechanical strength. Any oozing/seepage through the grafts was quantified as the volume of leakage per unit area of the graft in unit time.

\section{Bench testing of biocompatibility at static and dynamic conditions}

In-vitro biocompatibility was tested by seeding human umbilical cord vein endothelial cells (HUVECs) described in the Supplemental file. Briefly, we undertook static cell culture over $72 \mathrm{~h}$ on small nanotextiles samples aseptically transferred to 96-well plate and seeded with $2 \times 10^{4}$ HUVECs. Next, the samples were washed with phosphate-buffered saline (PBS), fixed with $4 \%$ paraformaldehyde (PFA), washed in PBS, dehydrated in gradient ethanol, and imaged using SEM. We then undertook cell cultures on the whole NanoGraft to assess cytotoxicity and amount of engrafted cells. First, HUVECs $\left(10^{5}\right.$ cells) were seeded in the NanoGraft at static conditions by placing them in a petri dish for $24 \mathrm{~h}$. Next, the NanoGraft was mounted in the bioreactor primed with culture media and run at physiological conditions (pressure $120 \mathrm{mmHg}, \sim 72$ cycles/min) for additional 24 and $48 \mathrm{~h}$. Then, the NanoGraft was cut into $5 \mathrm{~mm}$ segments. Cell presence and viability were tested using the Alamar blue assay, as reported in the Supplemental file. The number of viable cells remaining adherent in each segment of the NanoGraft was obtained from the standard curve. The percentage of adherent cells was calculated by comparing it with static control.

\section{In-vivo feasibility and mechanisms of engraftment}

Upon establishing the in-vitro mechanical safety of the NanoGraft in the dynamic bioreactor study, we undertook the in-vivo feasibility trial in an advanced porcine carotid artery replacement model. The in-vivo feasibility trial was conducted at the Translational Biomedical Research Centre (TBRC) for the large animal at the University of Bristol, Bristol, UK.

The animal procedures were in line with the U.K. Home Office regulations (Animal Act 1986) and were undertaken under a Project Licenses (PPL 30/3064 and PPL: 30/2854) granted by the Home Office after formal review and approval by the University of Bristol Animal Welfare and Ethics Review Body (AWERB). We used female Yorkshire pigs (approx. $60 \mathrm{~kg}$ ) receiving daily aspirin $(300 \mathrm{mg}$ ) with food. The procedure was in line with established approaches by our group [20]. General anaesthesia was achieved with IV $0.2 \mathrm{mg} / \mathrm{kg}$ morphine and Propofol. Mechanical ventilation was maintained with isoflurane in oxygen/air. Activated clotting time $(\mathrm{ACT})>400 \mathrm{~s}$ was maintained with Heparin (10,000-15,000 I.U.) and monitored every 15 min. During the surgical procedure, a continuous infusion of fentanyl five $\mu \mathrm{g} / \mathrm{kg} / \mathrm{hr}$ was administered along with $0.9 \%$ saline $(4 \mathrm{ml} / \mathrm{kg} / \mathrm{hr})$. After soft vascular clamping $1.5 \mathrm{~cm}$ segment of the native carotid artery was excised and $1.8-2.00 \mathrm{~cm}$ long grafts, either the pre-clotted Nanograft $(n=3+3)$ or clinical grade ePTFE for control $(n=3)$, were implanted via end-to-end anastomosis using polypropylene $7-0$ sutures (Prolene ${ }^{\circledR}$, Ethicon, USA). Animals were kept for 2 -week followed by termination under general anesthesia. Three additional NanoGrafts were implanted for 4-weeks to prolong the period of observation. 


\section{In-vivo vascular ultrasound doppler}

In-vivo vascular ultrasound doppler (USD) was acquired before the implant, 5 min after implant, 2-week for three Nanografts and three ePTFE grafts, and 4-week for three additional NanoGrafts. The USD was acquired under general anesthesia and continuous hemodynamic and surface electrocardiographic monitoring. Lumen size and blood flow velocity were measured and compared within groups and across groups. The inner diameter and intimal thickening of the proximal and distal anastomosis and mid graft portion were determined by 2D Doppler (MySono U6, Samsung, Korea). Blood flow through the implanted graft was determined both qualitatively and quantitatively by using the color Doppler mode.

\section{Ex-vivo optical coherence tomography-OCT}

All the carotid arteries inclusive of all the grafts were explanted at 2 and 4 weeks under general anesthesia at termination and placed in $0.9 \%$ saline solution. Next, the lumen area of the excised grafts was assessed using OCT (Zeiss, Germany), with an ex-vivo pullback being performed throughout the length of each specimen.

\section{Histological evaluation}

More detailed methods are presented in the Additional file 1 . All the samples were stored at $4{ }^{\circ} \mathrm{C}$ in PBS after fixing in $10 \%$ Neutral Buffered Saline (NBF) for 24 h. Samples were embedded in paraffin, and sections of $5 \mu \mathrm{m}$ thickness were assessed for histological findings.

\section{Hematoxylin and Eosin staining}

Samples were stained with Hematoxylin (Sigma Aldrich, USA) for $2 \mathrm{~min}$, then rinsed in distilled water for $3 \mathrm{~min}$. Sections were stained using 0.5\% Eosin (Sigma Aldrich, USA), washed for $3 \mathrm{~min}$ in tap water, dehydrated in 100\% ethanol, rinsed in xylene for $5 \mathrm{~min}$, and imaged using Leica compound microscope (DM500, Germany).

\section{Verhoeff-Van Gieson Stain for elastin}

Sections were stained with Verhoeff's solution (Sigma, USA) as detailed in the Supplementary file and checked microscopically for elastin fibers. The slides were treated with $5 \%$ sodium thiosulfate and washed in running tap water prior to counterstaining with Gieson's solution for 3-5 min. The specimens were dehydrated quickly with alcohol and mounted with coverslips using DPX mountant (Sigma Aldrich, USA).

\section{Masson's Trichrome Staining for collagen}

The slides were prepared as described in the Additional file 1 and stained in 1\% Biebrich scarlet-acid fuchsin solution for $10 \mathrm{~min}$ (Sigma, USA). Tissue was differentiated in the phosphomolybdic-phosphotungstic acid solution for $10 \mathrm{~min}$, and the slide transferred into Light green $2 \%$ solution, followed by a wash in distilled water.

\section{Alcian blue (acid mucosubstances)}

The slides were stained in a $1 \%$ alcian blue solution with $3 \%$ acetic acid (Sigma, USA) for $30 \mathrm{~min}$, washed in running tap water for $2 \mathrm{~min}$, and counterstained in the nuclear fast red solution for $5 \mathrm{~min}$.

\section{Immuno-histofluorescence}

Samples were fixed in $10 \%$ NBF, washed twice with PBS for the $30 \mathrm{~s}$, and tissue exposed to $200 \mu \mathrm{l}$ of permeabilization buffer (0.5\% Triton-X-100, Sigma Aldrich, USA) for 10-15 min. Next, additional PBS washing, incubation with $1 \mathrm{mg} / \mathrm{ml}$ Bovine Serum Albumin (Sigma Aldrich, USA) for $1 \mathrm{~h}$ and exposed to antibody solutions in sequence (primary antibody solution, secondary antibody solution streptavidin Alexa-488, and anti-alphasmooth muscle-Cy3 antibody) as described in Additional file 1.

\section{Results}

In-vitro testing of leakage, physical properties, and biocompatibility of the single layered NanoGraft

Flexible and kink-resistant nanotextile conduits of $4 \mathrm{~mm}$ diameter were developed from polymeric nanofibrous yarns of electrospun PLLA using a modified weaving system as described earlier [18], see Fig. 1a-c. Longitudinal and circumferential yarns were tightly interwoven to create the nanotextile conduit with low porosity, as shown in Fig. 1c (inset). This interwoven structure resulted in a significantly high burst pressure compared to the control ePTFE graft $(\mathrm{p}<0.0001)$ (Fig. 1d). Minimal leakage is an essential requirement for engineered vascular grafts to reach preclinical/clinical applications. To achieve this, the single layered prosthesis was pre-clotted with blood. Bench testing in a dynamic bioreactor (Fig. 1d-f) showed that the non-clotted NanoGraft had marked water leakage when subjected to arterial pulsatile pressures (Fig. 1d). However, the pre-clotted NanoGraft showed significantly reduced water entry pressure and permeability compared to the non-clotted conduit (Fig. 1e-f; both $\mathrm{p}<0.0001)$, which was similar to the water entry pressure and permeability observed for ePTFE control grafts (Fig. 1e-f). Other mechanical properties such as radial stiffness, suture retention, and tensile strength were superior for the NanoGraft (Additional file 1: Figure S1). Thus, despite being single layered, the NanoGraft could satisfy all the vital attributes for a vascular arterial graft.

One key aspect of the in-vitro validation of the Nanograft before in-vivo testing was to assess its biocompatibility under arterial dynamic conditions. Specifically, it 


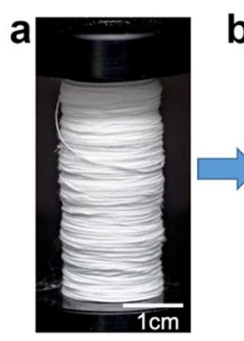

d
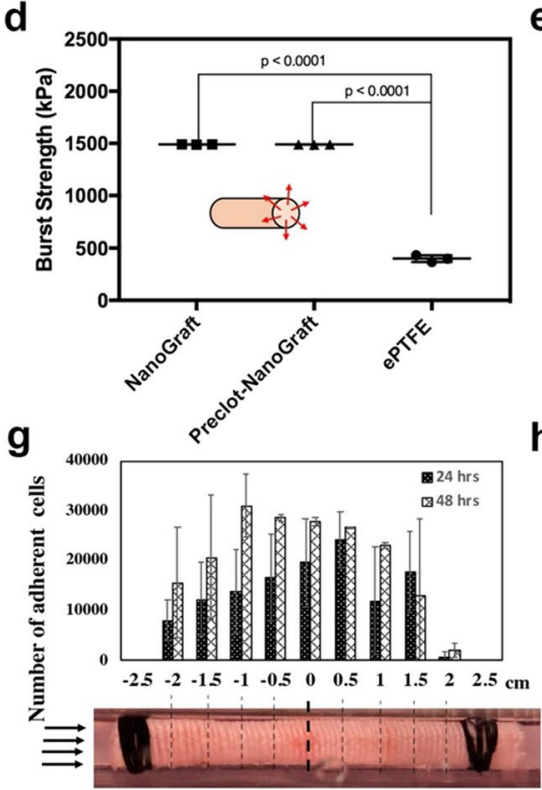

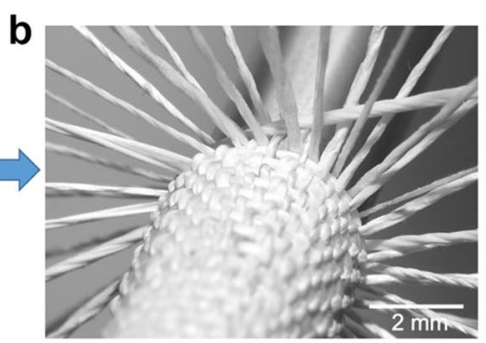

e

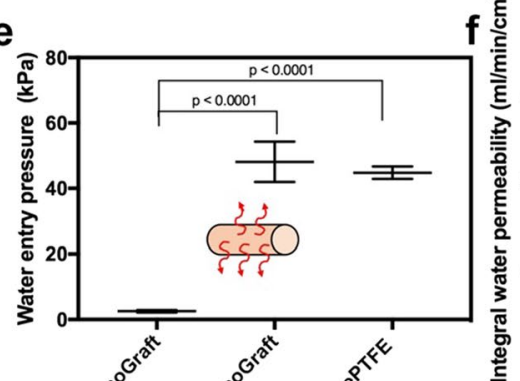

h

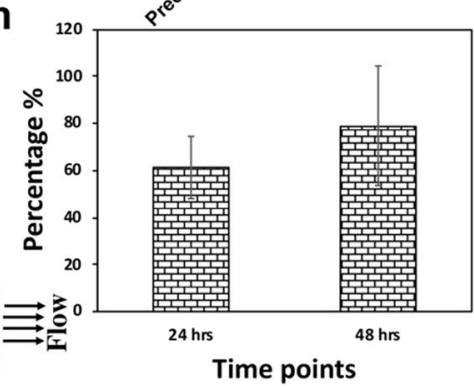

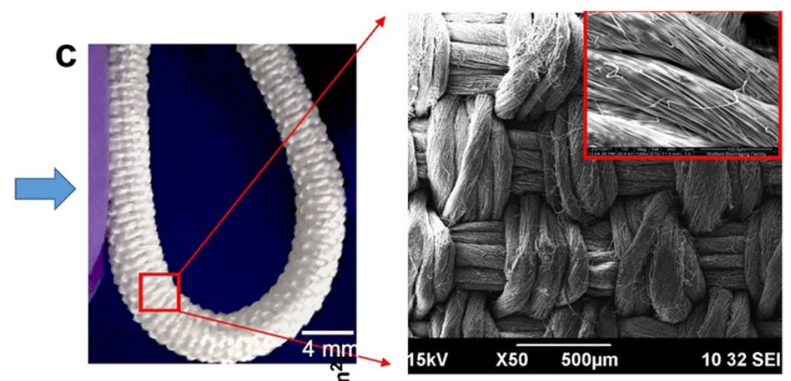

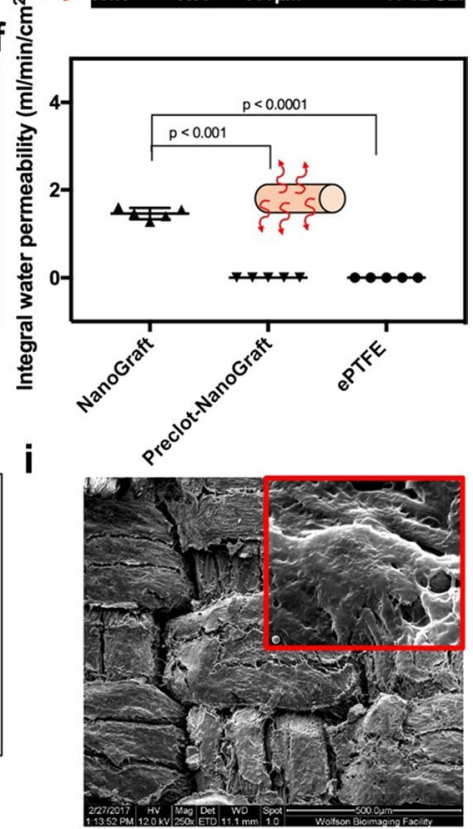

Fig. 1 Fabrication and in vitro characterization of the NanoGraft. a spool of nanofibrous yarns $\mathbf{b}$ fabrication of tightly packed woven conduits using multiple electrospun yarns c optical image of the NanoGraft (inset shows the SEM micrographs of the nanotextile) $\mathbf{d}$, e physical properties of NanoGraft compared to the commercial ePTFE graft $\mathbf{g}$-i adherence of HUVECs on the NanoGraft under dynamic flow conditions $\mathbf{g}$ Alamar assay showing cell distribution in the NanoGraft $\mathbf{h}$ the percentage of cells adhered on the NanoGraft under dynamic conditions for 24 and $48 \mathrm{~h} \mathbf{i}$ SEM micrographs of the NanoGraft showing cell coverage

was important to assess the endothelialisation potential of this nanotextile conduit. The Alamar blue assay demonstrated the presence of a large number of viable endothelial cells adherent to the NanoGraft and distributed uniformly at 24 and $48 \mathrm{~h}$ throughout its length, with the exception at both ends where the cannula was inserted to secure the graft to the flow circuit (Fig. 1gh). Noticeably, the number of cells increased from 24 to $48 \mathrm{~h}$, indicating cell expansion/proliferation (Fig. 1h). This experiment confirmed that the NanoGraft permitted rapid endothelial cell attachment ((Fig. 1g) and its firm engraftment, given the minimal cell detachment under dynamic arterial conditions (Fig. 1i). This biocompatibility might be due to the superhydrophilicity of the material used, which allows for faster endothelial adhesion, thereby promoting proliferation [21-23].

\section{In-vivo feasibility testing}

Having validated in-vitro the physical properties and biocompatibility of the NanoGraft, we then undertook an in-vivo feasibility trial of pre-clotted NanoGrafts, using clinical-grade ePTFE grafts as control for the 2-week time point. The range of internal diameter of the NanoGrafts was 3.6-3.9 mm. Both NanoGraft and ePTFE grafts exhibited good surgical suturability and handling properties during anastomosis and had a similar vascular cross-clamping time of 12-15 min (Fig. 2a). Additionally, NanoGraft exhibited no signs of fraying at the edges during anastomosis (Additional file 3: Video 2). However, ePTFE grafts showed significant suture line oozing after the restoration of arterial blood flow through the graft (Additional file 1: Figure S2 and Additional file 4: Video 3), which was not observed with NanoGrafts (Additional 

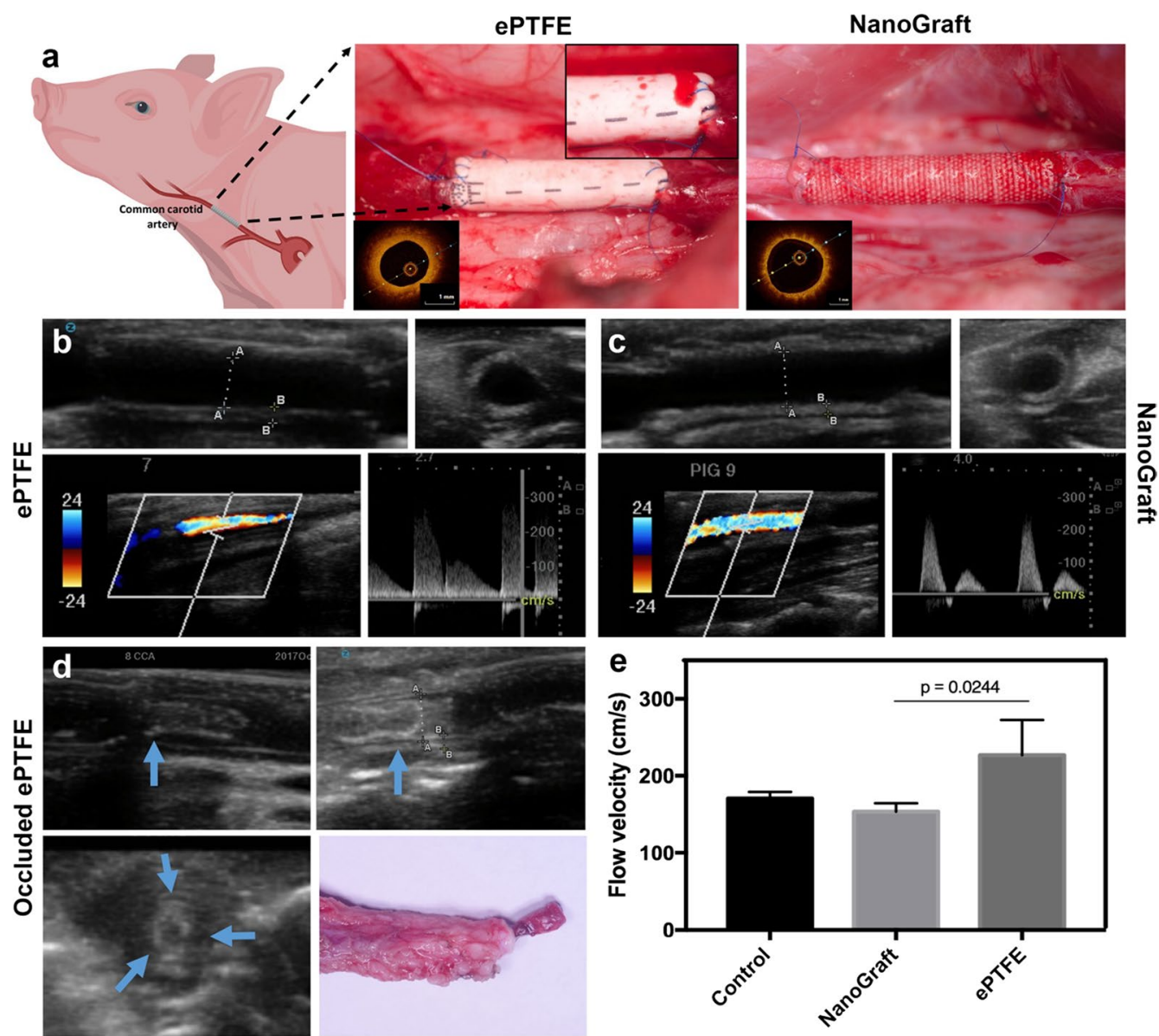

Fig. 2 In-vivo implantation and imaging assessment of ePTFE and NanoGraft grafts: a direct representative picture of the ePTFE graft with oozing from suture line and NanoGraft with no oozing. Inset shows the OCT image of the patent grafts. Representative 2D Doppler of the longitudinal and cross-sectional view of $\mathbf{b}$ ePTFE and $\mathbf{c}$ NanoGrafts (distance A- $3.8 \mathrm{~mm}, \mathrm{~B}-0.7 \mathrm{~mm}$ ), with the corresponding qualitative and quantitative evaluation of blood flow through the grafts (bottom in $\mathbf{b}$ and $\mathbf{c}$ ). $\mathbf{d}$ 2D Doppler of the longitudinal and cross-sectional view of the occluded ePTFE grafts (blue arrows depict the regions with adherent clots) and its corresponding optical image. e Quantitative evaluation of blood flow through the patent synthetic grafts before and after implantation

file 5: Video 4). The anastomotic oozing of the ePTFE grafts resolved 7-10 min after reversing the effect of Heparin with protamine sulfate. All intended procedures were completed successfully and reached the predefined termination point, with no animals being excluded.

\section{In-vivo vascular Doppler and ex-vivo OCT imaging}

USD results showed that the synthetic grafts had an average wall thickness of $400-500 \mu \mathrm{m}$, similar to the native carotid artery. All the grafts were patent soon after the surgical implantation. Late graft patency, lumen size, and blood flow velocity are shown in Fig. 2b, c using 2D imaging (longitudinal and transverse) as well as by qualitative and quantitative assessment of blood flow. In-vivo USD imaging just before termination confirmed 100\% patency of the NanoGraft at 2-weeks (3/3) (Additional file 6: Video 5) and 4-weeks (3/3), compared to $67 \%$ $(2 / 3)$ for ePTFE control at 2-weeks. One ePTFE graft was occluded at this time point, as shown in Fig. 2d. In addition, at 2-weeks, there was a higher blood flow velocity in ePTFE grafts compared to NanoGrafts and native carotid artery controls $(p=0.02$; Fig. 2 e). Ex-vivo OCT imaging was conducted post-termination, confirming the early patency of the implanted grafts (see representative images in Fig. 2a inset), correlating with the patency data seen with in-vivo ultrasound Doppler (Additional file 7: Video 6).

\section{Mechanisms of engraftment, inflammatory response, and vascular remodeling}

Preliminary histology showed no signs of gross thrombi on the luminal surface of the NanoGrafts (Fig. 3a) in 

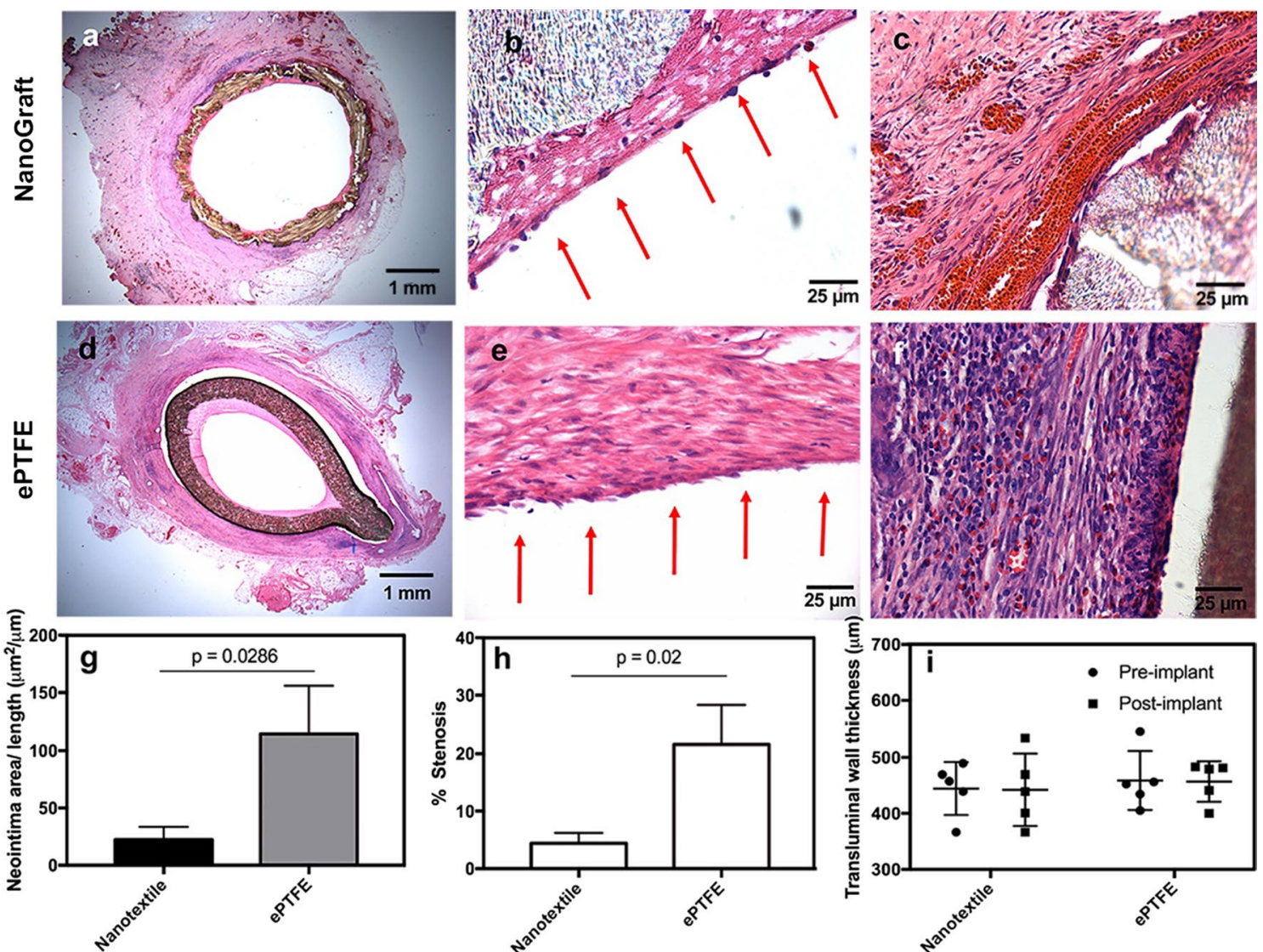

Fig. 3 Histological and histomorphometry evaluation of the implanted synthetic grafts after 2 weeks of implantation. H\&E staining of the midsection of NanoGraft a entire graft Sect. $(1.25 \times)$ b cell lining on the luminal surface of NanoGraft (depicted by red arrows) c abluminal surface of the graft with a minimal inflammatory response). $\mathbf{d}-\mathbf{e}$ H\&E staining of the midsection of ePTFE grafts $\mathbf{d}$ entire graft Sect. (1.25 $\times$ ) $\mathbf{e}$ cell lining on the luminal surface of ePTFE (depicted by red arrows) $\mathbf{f}$ abluminal surface of the graft with high inflammatory cells $\mathbf{g}$ Neointimal area $\mathbf{h}$ Percentage of graft stenosis and $\mathbf{i}$ Transluminal wall thickness, of the implanted synthetic grafts. Statistical significance between the two groups was assessed using a paired t-test. Error bars represent standard deviation. P-value of each comparison is depicted in the plot

contrast to one of the ePTFE grafts, which was entirely occluded by thrombus formation (Fig. 2d and Additional file 4: Figure S3), despite the use of standard platelet inhibition with aspirin. Closer histological evaluation showed neointimal thickening and early endothelialisation in both the grafts (Fig. 3b and e). However, intimal thickening for the NanoGrafts was significantly less than ePTFE grafts $(22.39 \pm 11.19$ vs. $114.71 \pm 41.20 ; \mathrm{p}=0.028$; Fig. $3 g$ ). No evidence of changes in the transmural wall thickness was observed at 2-weeks for NanoGraft and ePTFE grafts, as shown in Fig. 3i, demonstrating the mechanical integrity of the grafts. The outer diameter of the grafts also did not show any alterations pre and post-implantation, suggesting the absence of late aneurysm or graft rupture. While these early observations are encouraging, this essential aspect needs further validation in long-term in-vivo preclinical studies. Extracellular matrix deposition (ECMD) was observed in the void spaces mainly across the walls of the NanoGraft, between longitudinal and circumferential yarns, with no impact on the lumen size. Conversely, the ePTFE grafts showed a stenotic and non-homogeneous ECMD with neointimal formation, which reduced the lumen by $30-40 \%$ compared to baseline ( $p=0.02$; Fig. $3 \mathrm{~d}$, e and h). Inflammatory response observed at the interstitial interface of the NanoGraft was minimal, with excellent tissue integration. (Fig. 3c). In contrast, ePTFE grafts showed a severe inflammatory reaction in the peri-graft region, with a substantial number of neutrophils, lymphocytes and macrophages and no cellular integration (Fig. $3 \mathrm{f}$ and Additional file 1: Figure S4).

Finally, the intimal thickening observed in the NanoGraft was minimal, with uniform and complete endothelial coverage which can be attributed to its transluminal capillary in-growth as illustrated in Fig. 4a. However, this endothelial lining was patchy in the ePTFE graft. 

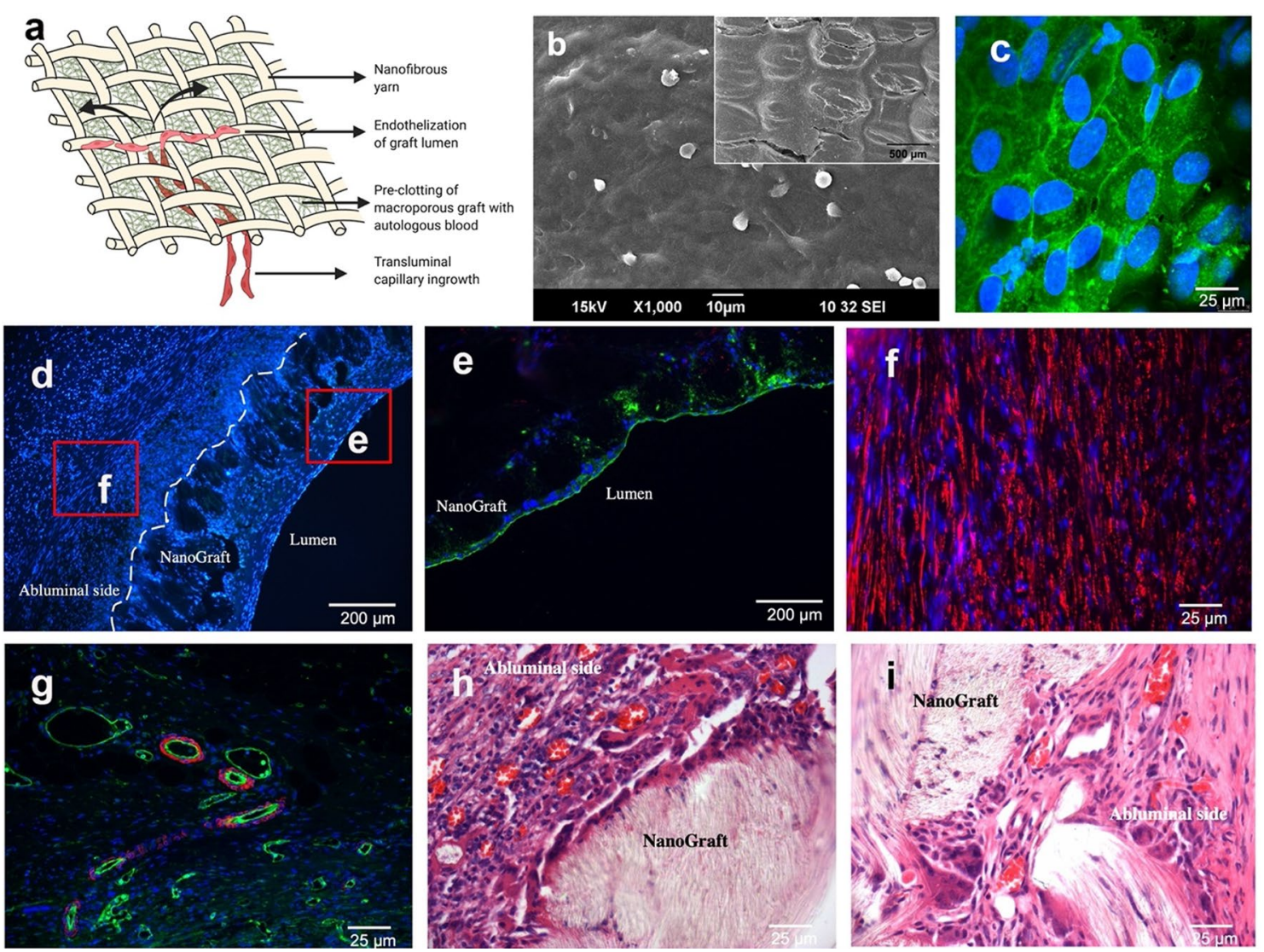

Fig. 4 Transmural endothelialisation in biodegradable vascular NanoGraft. a Illustration of transmural capillary in-growth through the porous woven structure of the nanotextile graft. $\mathbf{b}$ SEM micrograph showing endothelial coverage on the entire surface of the graft (inset low magnification). Confocal images of the explanted NanoGraft c en face staining on the luminal surface of the NanoGraft showing complete endothelial coverage with tight junctions $\mathbf{d}$ cross-sectional view of vascular graft showing an abundance of infiltrated cells stained for nuclei (DAPI-blue) e Immunohistofluorescence staining of the mid-portion of NanoGraft. Tissues stained for nuclei-DAPI (blue), Endothelial cells_-Wheat germ agglutinin (green). f Presence of circumferentially aligned smooth muscle cells (alpha-smooth muscle actin-red) on the abluminal surface of the graft. $\mathbf{g}$ Neocapillaries formed at the abluminal side of NanoGraft $(400 \times)$. H\&E staining shows neocapillary in-growth in the $\mathbf{h}$ abluminal and $\mathbf{i}$ luminal regions of the porous nanotextile graft

This finding was confirmed by the en face staining of the endothelial surface of NanoGrafts, as shown in Fig. 4c and Additional file 8: Video 7, and further by SEM micrographs (Fig. 4b). High magnification immunostaining endorsed the formation of tight endothelial junction as shown in Additional file 1: Figure S5. Also, both synthetic grafts showed a large amount of circumferentially oriented smooth muscle cells, as shown in Fig. $4 \mathrm{~d}$ and $\mathrm{f}$ with numerous neo-capillary vessels in the luminal and abluminal regions of the NanoGraft as shown in Fig. 4g-i.

A significant transmural neo-capillary formation was observed in NanoGrafts at 4-weeks after surgery, via hematoxylin \& eosin staining (Fig. 5a, i-iv). Besides, qualitative and quantitative evaluation of ECMD for collagen, elastin, and mucopolysaccharides revealed an appreciable amount of collagen secretion from the fibroblast/smooth muscle cells, as evident from Masson trichome staining (Fig. 5b, i-iv). Van Gieson stain confirmed elastin deposition at 4-weeks compared to 2-weeks from implantation (Fig. 5c, i-iv). Finally, a higher amount of mucopolysaccharides (Fig. 5d, i-iv) was measured in the NanoGrafts at 4-weeks after surgical implantation, whose absence in synthetic grafts has been reported previously to cause hypercoagulability [24].

\section{Discussion}

Biodegradable grafts have attracted recent interest due to their inherent regenerative potential and ability to resorb over time, eliminating life-long foreign-body inflammatory activation [25-28]. Manufacturing techniques have included electrospinning, casting, and freeze drying $[15,16]$. Electrospinning, resulting in nano/micro-scale 

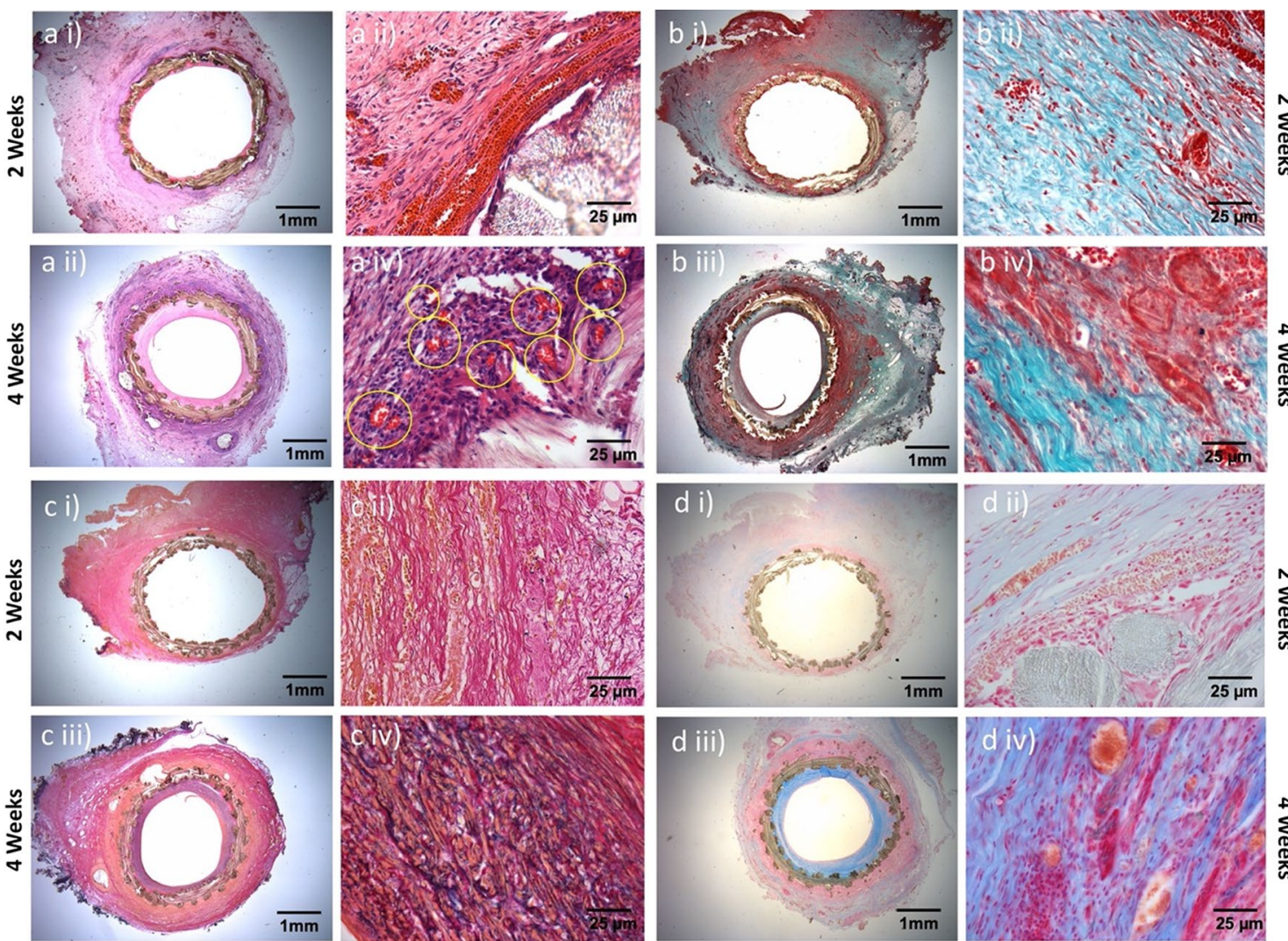

Fig. 5 Histological analysis of the NanoGraft showing an increase in neocapillary formation, elastin, collagen, and glycosaminoglycans after 2 and 4 weeks of implantation. a H\&E staining b Masson-Trichrome staining for collagen (bluish-green) $\mathbf{c}$ Verhoeff-Van Gieson staining for elastin content (blue-black) and $\mathbf{d}$ Alcian blue stain for mucopolysaccharide (light blue)

fibers, has been the most widely used approach in this field so far [27]. Various polymers individually or in combination (PCL, PLLA, PGA, gelatin) and in diverse configurations (aligned, random, hybrid) have been investigated for this purpose [27-29]. Scaffolds fabricated with fibers in this dimension and orientation mimics the extracellular matrix of the native artery, thereby promoting rapid endothelisation, reduced thrombogenicity, cellular infiltration, and regeneration of the polymeric graft [15]. In addition, nano/micro-scale fibers offer better elastic properties, conferring flexibility and manoeuvrability to the vascular graft [29]. However, no biodegradable small arterial grafts have yet been approved by the FDA due to their low burst strength, suture retention, lack of flexibility and potential for kinking, and thereby its inability to meet the essential requirements of the relevant ISO standards 7198:2016 [30, 31]. To address these limitations, we fabricated a vascular graft from bundles of nanofibrous threads called nanoyarns [17] by the textile technique of weaving [18]. This woven nanotextile graft was rendered impervious by a conventional clinical pre-treatment with autologous blood [19], which sealed off the graft interstices by controlled fibrin formation. Excessive heparin containing blood was flushed in the graft lumen to dislodge any surface clots and neutralize unreacted thrombin. Leak test was performed according to the ISO standards and also at physiological pulsatile flow conditions along with high arterial pressure up to $400 \mathrm{~mm} \mathrm{Hg}$. Biodegradable grafts fabricated by conventional nanofiber techniques are highly susceptible to mechanical failure at higher arterial pressure, which is common during the post-operative recovery phase, leading to graft failure [32]. In contrast, the NanoGraft revealed high mechanical strength and burst pressure, which can be attributed to the hierarchal arrangement of interwoven nanofibrous yarns along the longitudinal and circumferential directions. Similarly, suture retention of grafts depends greatly on fiber orientation [33]. It is the interwoven pattern utilised in the NanoGraft which conferred it with high suture retention strength compared to the non-woven form [18] and commercial ePTFE grafts (see Additional file 9: Video 8).

In-vivo feasibility studies were conducted at clinical standards, as this is of paramount importance to 
ascertain the safety and pre-clinical efficacy of the device to enhance its translation potential. Biodegradable grafts must withstand the pressures exerted by pulsatile flow without bursting or experiencing permanent and dangerous deformation through aneurysm or tearing. At the same time, colonization of the graft by inflammatory cells may trigger structural weaknesses. Noticeably, thrombus formation within the grafts can be mitigated if alterations in blood flow velocity across the graft length are minimal, and the luminal graft surface characteristics are nonthrombogenic [34]. In addition, the graft should possess suitable mechanical characteristics to prevent the formation of high shear stress at the anastomotic sites and be of a geometry that does not trigger detrimental flow patterns, as both of these essential factors are associated with graft failure [35]. The formation of micro-thrombi has previously been associated with ePTFE grafts [36]. Also, occlusion of ePTFE grafts has been reported with overall patency of $67 \%$ at 1 -month when implanted in femoropopliteal position [37] or $<50 \%$ when implanted as an arteriovenous graft in the porcine carotid model [38]. Based on this preclinical work and our findings indicating the high rate of graft occlusion at 2-weeks, ePTFE implantation was restricted in our study to 2 weeks only (see Additional file 10: Video 9).

The excellent in-vivo biocompatibility of NanoGraft is an essential factor that may facilitate longevity, which was not observed in ePTFE grafts at the same time point. This observation might be associated with the uniform transmural neo-capillary formation within the walls of the NanoGraft, resembling vasa-vasorum, which was absent in ePTFE grafts. The formation of these transmural neo-capillaries may be attributed to the porous nature of the NanoGraft. Similar observations have been made by others related to graft porosity $[39,40]$. Additionally, pre-clotting of the NanoGraft would result in a fibrin plug that makes the graft impervious to blood flow. There is ample evidence in literature which suggests enhanced neo-capillary formation in the presence of fibrin [41], supporting our observation. Previous studies have shown endothelial coverage on synthetic graft surfaces to be enhanced by transmural endothelialisation through neocapillary in-growth from the perivascular region. This process is reported to occur predominantly in grafts with sufficient porosity [39, 40, 42]. Hence, it might be argued that the spontaneous endothelialisation observed in the NanoGraft may be triggered by the presence of fibers in the nanoscale, coupled with the graft porosity. One possible explanation for the observed rapid and uniform endothelial coverage in the NanoGraft is the enhanced adsorption of serum proteins on the superhydrophilic nanotextile [18], which facilitates cellular adhesion and subsequent proliferation in the luminal graft surface.
However, a detailed mechanistic study is required to probe and understand the exact mechanisms of engraftment and healing in-situ, wherein the endothelization can also be facilitated or derived from circulating endothelial progenitor cells or migration of endothelial cells from in-situ arteries across anastomotic sites [43].

Synthetics grafts like ePTFE and Dacron ${ }^{\circledR}$ are incapable of spontaneous endothelialisation on the luminal surface of the graft $[22,44]$. This is mainly ascribed to the hydrophobic nature of these graft materials, which hinders endothelialisation, and also leads to platelet adhesion and thrombosis. On the contrary, the unique architecture of the nanotextile renders the luminal surface of the NanoGraft superhydrophilic, owing to the aligned nanofibres in the electrospun yarn, which in turn makes the surface antithrombotic.

The deposition of extracellular matrix components such as elastin, collagen and mucopolysaccharides would also provide mechanical integrity during the resorption of NanoGraft. Elastin is expected to increase only after the loss of mechanical integrity of the synthetic graft, observed typically in long-term in-vivo studies. Collagen improves the mechanical resilience [45], while elastin imparts elastic recoil and preserves the structural integrity when exposed to pulsatile flow conditions [46-48]. An increase in collagen and elastin from 2 to 4 weeks shows a promising translational potential of the biodegradable NanoGraft.

\section{Conclusion}

The study confirmed the in-vivo feasibility and safety of the single-layered vascular prosthesis, viz., NanoGraft. Its use appears to be associated with increased transmural in-growth of host cells and neo-capillaries, complete neo-endothelial coverage, patency, and reduced neointimal thickening when compared to ePTFE, although more data is needed to confirm this. Suturability and surgical handling were as good as ePTFE grafts. Physical properties of the NanoGraft were in concurrence with ISO standards, with no dilatation/aneurysm formation observed at 4-week. A pivotal porcine long-term preclinical study is warranted to confirm the safety/efficacy of the proposed graft.

\section{Supplementary Information}

The online version contains supplementary material available at https://doi. org/10.1186/s12951-022-01268-1.

Additional file 1: Materials and Methods, Figure S1. Mechanical properties of NanoGrafts a) High radial stiffness to resist distortion and compression b) Superior suture retention that demonstrates resistance to wear and tear edges c) Adequate tensile strength to prevent graft rupture. Figure S2. ePTFE grafts showed significant suture line oozing after the restoration of arterial blood flow following anastomosis with 7-0 sutures. 
Figure S3. The histopathological finding of the midsection of grafted ePTFE at 2 weeks a) total thrombotic occlusion at the luminal region b and c) higher magnification showing intact fibrin-clot at the graft interface. Figure S4. The histopathological finding of the midsection of grafted ePTFE at 2 weeks showing dense infiltration of immune cells in the abluminal section characterised by presence of lymphocytes (white arrows), macrophages (green arrow) and neutrophils (yellow arrows). Figure S5. En face immunofluorescence staining of NanoGraft at 2 weeks showed tight endothelial junctions (green -agglutinin on Endothelial, blue- DAPI nucleus).

Additional file 2: Video 1. Physiological properties of the NanoGraft were evaluated under arterial pulsatile flow conditions using a bioreactor (TGT DynaGen ${ }^{\circledR}$ Series, USA) primed with heparinized porcine blood.

Additional file 3: Video 2. Nanotextile based graft showed excellent suturability and lack of fraying at the edge.

Additional file 4: Video 3. ePTFE grafts showed significant suture line oozing after the restoration of arterial blood flow through the graft wall.

Additional file 5: Video 4. Lack of postoperative suture line bleeding and transmural blood leakage of NanoGraft.

Additional file 6: Video 5. Percutaneous ultrasound shows the luminal patency and pulsatile blood flow through the Nanograft at 2 weeks.

Additional file 7: Video 6. Percutaneous ultrasound shows the luminal patency and pulsatile blood flow through the ePTFE graft at 2 weeks.

Additional file 8: Video 7. Percutaneous ultrasound shows the total occlusion of ePTFE graft at 2 weeks.

Additional file 9: Video 8. Ex-vivo OCT post-termination confirmed the patency of Nanograft at 2 weeks.

Additional file 10: Video 9. En face staining of NanoGrafts confirms complete endothelial coverage at two weeks of post-implantation.

\section{Acknowledgements}

Joseph acknowledges the Commonwealth Scholarship Commission for the Split-site scholarship (INCN-2016-176) held with Prof. George at the University of Bristol, United Kingdom, and CSIR for Senior Research Fellowship (9/963(0035)2k14-EMRI). We thank the staff at the University of Bristol Translational Biomedical Research Centre, a UK national research facility for large animal co-funded by the British Heart Foundation and the Medical Research Council. In addition, we acknowledge Amrita Vishwa Vidyapeetham for infrastructural support.

\section{Authors' contributions}

$\mathrm{JJ}$ : developed the graft in Amrita, did lab-based work in Bristol, analyzed data and drafted the manuscript; VDB: assisted animal surgery in Bristol and undertook vascular doppler; NS: helped with lab-based work in Bristol and the bioreactor; AW: helped with graft engraftment lab-based work in Bristol; TWJ: undertook the ex-vivo optical coherence tomography on explanted grafts in Bristol; HMB: undertook mechanical strength work at Amrita and prepared Figs. 2a, 4a, and graphical abstract; PKV: helped developing the graft in Amrita before studies in Bristol; RJ: helped developing the graft in Amrita before studies in Bristol; SVN: helped developing the graft in Amrita and securing previous funding from the Indian Government; DM: helped with developing the graft in Amrita including supervising the Amrita-based bench testing and related interpretation, co-secured the funding for the Commonwealth Scholarship Commission for the Splitsite scholarship, co-drafted the manuscript; SJG: supervised the lab-based histology work in Bristol related to graft characterization and interpretation, co-secured the funding for the Commonwealth Scholarship Commission for the Split-site scholarship, co-drafted the manuscript; RA: acted as senior author, conceived and led all the bioreactor and in-vivo work done in Bristol and related interpretation, secured all funding to support bioreactor and in-vivo work in Bristol, co-drafted the manuscript and was corresponding author. All authors read and approved the final manuscript.

\section{Funding}

The bench-testing in a dynamic bioreactor and all the in-vivo feasibility studies presented in this manuscript were supported by grants awarded to Prof.
Ascione: the British Heart Foundation (BHF) (BHF IG/14/2/30991, and BHF/ $\mathrm{PG} / 16 / 104 / 32652)$, the University of Bristol Alumni, and the Medical Research Council (MRC) (MRC MR/L012723/1). The development of the NanoGraft technology at Amrita was funded by Department of Science and Technology (DST), Government of India, through the "Thematic Projects in Frontiers of Nanoscience \& Technology" (SR/NM/TP-15/2016G). The laboratory assays part of this study was supported by the Commonwealth Scholarship Commission, United Kingdom (INCN-2016-176).

\section{Availability of data and materials}

All the raw data used to generate this article is stored on the University of Bristol and Amrita servers with security access. We are happy to make the raw data available if necessary. We have extra aliquots of histology samples available if required. All the pre-clinical research files/daily medical notes for each experiment from surgery, critical care, and maintenance up to the termination are stored under secure access in the Bristol TBRC facility and can be made available to the Editorial Office at any time if requested.

\section{Declarations}

\section{Ethics approval and consent to participate}

This study did not involve patients and therefore no ethical approval or consent to participate was needed.

\section{Consent for publication}

All the authors have read this manuscript and given their consent to publication.

\section{Competing interests}

The authors declare no competing financial interests.

\section{Author details}

${ }^{1}$ Bristol Heart Institute and Translational Biomedical Research Centre, Faculty of Health Science, University of Bristol, Bristol BS2 8HW, UK. ${ }^{2}$ Centre for Nanosciences \& Molecular Medicine, Amrita Vishwa Vidyapeetham, Kochi 682041 India. ${ }^{3}$ Department of Cardiovascular and Thoracic Surgery, Amrita Institute of Medical Sciences \& Research Centre, Amrita Vishwa Vidyapeetham, Kochi 682 041, Kerala, India.

Received: 17 November 2021 Accepted: 16 January 2022 Published: 8 February 2022

\section{References}

1. Tomizawa Y. Vascular prostheses for aortocoronary bypass grafting: a review. Artif Organs. 1995;19:39-45. https://doi.org/10.1111/j.1525-1594. 1995.tb02242.x

2. Slovut DP, Lipsitz EC. Surgical technique and peripheral artery disease. Circulation. 2012;126:1127-38. https://doi.org/10.1161/CIRCULATIONAHA. 111.059048.

3. Seifu DG, Purnama A, Mequanint K, Mantovani D. Small-diameter vascular tissue engineering. Nat Rev Cardiol. 2013;10:410-21. https://doi.org/10. 1038/nrcardio.2013.77.

4. Klinkert P, Post PN, Breslau PJ, van Bockel JH. Saphenous vein versus PTFE for above-knee femoropopliteal bypass. A review of the literature. Eur J Vasc Endovasc Surg. 2004;27:357-62. https://doi.org/10.1016/j.ejvs.2003. 12.027 .

5. Rothuizen TC, Damanik FFR, Lavrijsen T, Visser MJT, Hamming JF, Lalai RA, Duijs JMGJ, van Zonneveld AJ, Hoefer IE, van Blitterswijk CA, RabelinkTJ, Moroni L, Rotmans J. Development and evaluation of in vivo tissue engineered blood vessels in a porcine model. Biomaterials. 2016;75:82-90. https://doi.org/10.1016/j.biomaterials.2015.10.023.

6. Roy-Chaudhury P, El-Khatib M, Campos-Naciff B, Wadehra D, Ramani K, Leesar M, Mistry M, Wang Y, Chan JS, Lee T, Munda R. Back to the future: how biology and technology could change the role of ptfe grafts in vascular access management. Semin Dial. 2012;25:495-504. https://doi. org/10.1111/j.1525-139X.2012.01091.x.

7. Gibson KD, Gillen DL, Caps MT, Kohler TR, Sherrard DJ, Stehman-Breen CO. Vascular access survival and incidence of revisions: a comparison of 
prosthetic grafts, simple autogenous fistulas, and venous transposition fistulas from the United States renal data system dialysis morbidity and mortality study. J Vasc Surg. 2001;34:694-700. https://doi.org/10.1067/ mva.2001.117890.

8. Parang P, Arora R. Coronary vein graft disease: pathogenesis and prevention. Can J Cardiol. 2009. https://doi.org/10.1016/S0828-282X(09)70486-6.

9. Burger DHC, Kappetein AP, Van Bockel JH, Breslau PJ. A prospective randomized trial comparing vein with polytetrafluoroethylene in above-knee femoropopliteal bypass grafting. JVasc Surg. 2000;32:278-83. https://doi. org/10.1067/mva.2000.106496.

10. Veith FJ, Gupta SK, Ascer E, White-Flores S, Samson RH, Scher LA, Towne JB, Bernhard VM, Bonier P, Flinn WR, Astelford P, Yao JST, Bergan JJ. Six-year prospective multicenter randomized comparison of autologous saphenous vein and expanded polytetrafluoroethylene grafts in infrainguinal arterial reconstructions. J Vasc Surg. 1986;3:104-14. https://doi.org/10. 1016/0741-5214(86)90073-X.

11. Yashar JJ, Thompson R, Burnard RJ, Weyman AK, Yashar J, Hopkins RW. Dacron vs vein for femoropopliteal arterial bypass: should the saphenous vein be spared? Arch Surg. 1981;116:1037-40. https://doi.org/10.1001/ archsurg.1981.01380200045009.

12. Ravari H, Kazemzade GH, Modaghegh MHS, Khashayar P. Patency rate and complications of polytetrafluoroethylene grafts compared with polyurethane grafts for hemodialysis access. Ups J Med Sci. 2010;115:245-8. https://doi.org/10.3109/03009731003678562.

13. Desai M, Seifalian AM, Hamilton G. Role of prosthetic conduits in coronary artery bypass grafting. Eur J Cardiothoracic Surg. 2011;40:394-8. https:// doi.org/10.1016/j.ejcts.2010.11.050.

14. Lemson MS, Tordoir JH, Daemen MJ, Kitslaar PJ. Intimal hyperplasia in vascular grafts. Eur J Vasc Endovasc Surg. 2000;19:336-50. https://doi.org/ 10.1053/ejvs.1999.1040.

15. Hiob MA, She S, Muiznieks LD, Weiss AS. Biomaterials and modifications in the development of small-diameter vascular grafts. ACS Biomater Sci Eng. 2017:3:712-23. https://doi.org/10.1021/acsbiomaterials.6b00220.

16. Xue L, Greisler HP. Biomaterials in the development and future of vascular grafts. J Vasc Surg. 2003;37:472-80. https://doi.org/10.1067/mva.2003.88.

17. Joseph J, Nair SV, Menon D. Integrating substrateless electrospinning with textile technology for creating biodegradable three-dimensional structures. Nano Lett. 2015;15:5420-6. https://doi.org/10.1021/acs.nanol ett.5b01815.

18. Joseph J, Krishnan AG, Cherian AM, Rajagopalan B, Jose R, Varma $P_{\text {, }}$ Maniyal V, Balakrishnan S, Nair SV, Menon D. Transforming nanofibers into woven nanotextiles for vascular application. ACS Appl Mater Interfaces. 2018;10:19449-58. https://doi.org/10.1021/acsami.8b05096.

19. Yates SG, Barros D'Sa AB, Berger K, Fernandez LG, Wood SJ, Rittenhouse EA, Davis CC, Mansfield PB, Sauvage LR. The preclotting of porous arterial prostheses. Ann Surg. 1978;188:611-22. https://doi.org/10.1097/00000 658-197811000-00005.

20. Sulaiman NS, Bond AR, Bruno VD, Joseph J, Johnson JL, Suleiman MS, George SJ, Ascione R. Effective decellularisation of human saphenous veins for biocompatible arterial tissue engineering applications: bench optimisation and feasibility in vivo testing. J Tiss Eng. 2021. https://doi. org/10.1177/2041731420987529.

21. Dekker A, Reitsma K, Beugeling T, Bantjes A, Feijen J, van Aken WG. Adhesion of endothelial cells and adsorption of serum proteins on gas plasmatreated polytetrafluoroethylene. Biomaterials. 1991;12:130-8. https://doi. org/10.1016/0142-9612(91)90191-C.

22. Sarkar S, Sales KM, Hamilton G, Seifalian AM. Addressing thrombogenicity in vascular graft construction. J Biomed Mater Res Part B Appl Biomater. 2007:82:100-8. https://doi.org/10.1002/jbm.b.30710.

23. Ma Z, Kotaki M, Yong T, He W, Ramakrishna S. Surface engineering of electrospun polyethylene terephthalate (PET) nanofibers towards development of a new material for blood vessel engineering. Biomaterials. 2005;26:2527-36. https://doi.org/10.1016/j.biomaterials.2004.07.026.

24. Philpott DE, Kato K. Mucopolysaccharide vascular coating relationship to environmental factors. In: Swenberg CE, Horneck G, Stassinopoulos EG, editors. Biological effects and physics of solar and galactic cosmic radiation. Boston: Springer; 1993. p. 217-26. https://doi.org/10.1007/9781-4615-2918-7_21.

25. Melchiorri AJ, Hibino N, Fisher JP. Strategies and techniques to enhance the in situ endothelialization of small-diameter biodegradable polymeric vascular grafts. Tissue Eng Part B Rev. 2013;19:292-307. https://doi.org/10. 1089/ten.teb.2012.0577.

26. Pektok E, Nottelet B, Tille JC, Gurny R, Kalangos A, Moeller M, Walpoth $\mathrm{BH}$. Degradation and healing characteristics of small-diameter poly $(\varepsilon-$ caprolactone) vascular grafts in the rat systemic arterial circulation. Circulation. 2008;1 18:2563-70. https://doi.org/10.1161/CIRCULATIONAHA. 108. 795732.

27. Hasan A, Memic A, Annabi N, Hossain M, Paul A, Dokmeci MR, Dehghani F, Khademhosseini A. Electrospun scaffolds for tissue engineering of vascular grafts. Acta Biomater. 2014;10:11-25. https://doi.org/10.1016/j. actbio.2013.08.022.

28. Cleary MA, Geiger E, Grady C, Best C, Naito Y, Breuer C. Vascular tissue engineering: the next generation. Trends Mol Med. 2012;18:394-404. https://doi.org/10.1016/j.molmed.2012.04.013.

29. Lee KH, Kim HY, Khil MS, Ra YM, Lee DR. Characterization of nano-structured poly( $\varepsilon$-caprolactone) nonwoven mats via electrospinning. Polymer (Guildf). 2003;44:1287-94. https://doi.org/10.1016/S0032-3861(02) 00820-0.

30. DIN EN ISO 7198, Cardiovascular implants and extracorporeal systemsVascular Prostheses-Tubular vascular grafts and vascular patches, ISO 7198. 24 (2014)

31. Stacy MR, Naito Y, Maxfield MW, Kurobe H, Tara S, Chan C, Rocco KA, Shinoka T, Sinusas AJ, Breuer CK. Targeted imaging of matrix metalloproteinase activity in the evaluation of remodeling tissue-engineered vascular grafts implanted in a growing lamb model. J Thorac Cardiovasc Surg. 2014;148:2227-33. https://doi.org/10.1016/j.jtcvs.2014.05.037.

32. Mine Y, Mitsui H, Oshima Y, Noishiki Y, Nakai M, Sano S. Suture retention strength of expanded polytetrafluoroethylene (ePTFE) graft. Acta Med Okayama. 2010;64:121-8. https://doi.org/10.18926/AMO/32846.

33. Meng $X$, Wang $X$, Jiang $Y$, Zhang B, Li K, Li Q. Suture retention strength of P(LLA-CL) tissue-engineered vascular grafts. RSC Adv. 2019;9:21258-64. https://doi.org/10.1039/C9RA04529E.

34. Begovac PC, Thomson RC, Fisher JL, Hughson A, Gällhagen A. Improvements in GORE-TEX ${ }^{\circledR}$ vascular graft performance by Carmeda ${ }^{\circledR}$ BioActive Surface heparin immobilization. Eur J Vasc Endovasc Surg. 2003;25:432-7. https://doi.org/10.1053/ejvs.2002.1909.

35. Ojha M, Cobbold RSC, Johnston KW. Influence of angle on wall shear stress distribution for an end-to-side anastomosis. JVasc Surg. 1994;19:1067-73. https://doi.org/10.1016/S0741-5214(94)70219-5.

36. Clowes AW, Gown AM, Hanson SR, Reidy MA. Mechanisms of arterial graft failure. 1. Role of cellular proliferation in early healing of PTFE prostheses. Am J Pathol. 1985;118;43-54.

37. Mrówczyński W, Mugnai D, De Valence S, Tille JC, Khabiri E, Cikirikcioglu M, Möller M, Walpoth BH. Porcine carotid artery replacement with biodegradable electrospun poly-e-caprolactone vascular prosthesis. J Vasc Surg. 2014;59:210-9. https://doi.org/10.1016/j.jvs.2013.03.004.

38. Rotmans JI, Velema E, Verhagen HJM, Blankensteijn JD, Kastelein JJP, De Kleijn DPV, Yo M, Pasterkamp G, Stroes ESG. Rapid, arteriovenous graft failure due to intimal hyperplasia: a porcine, bilateral, carotid arteriovenous graft model. J Surg Res. 2003;1 13:161-71. https://doi.org/10.1016/S00224804(03)00228-2.

39. Pennel T, Bezuidenhout D, Koehne J, Davies NH, Zilla P. Transmural capillary ingrowth is essential for confluent vascular graft healing. Acta Biomater. 2018;65:237-47. https://doi.org/10.1016/j.actbio.2017.10.038.

40. Clowes AW, Kirkman TR, Reidy MA. Mechanisms of arterial graft healing. Rapid transmural capillary ingrowth provides a source of intimal endothelium and smooth muscle in porous PTFE prostheses. Am J Pathol. 1986;123:220-30.

41. Ceccarelli J, Putnam AJ. Sculpting the blank slate: How fibrin's support of vascularization can inspire biomaterial design. Acta Biomater. 2014;10:1515-23. https://doi.org/10.1016/j.actbio.2013.07.043.

42. Contreras MA, Quist WC, LoGerfo FW. Effect of porosity on small-diameter vascular graft healing. Microsurgery. 2000;20:15-21. https://doi.org/10. 1002/(SICI)1098-2752(2000)20:1\%3c15\%:AID-MICR3\%3e3.0.CO;2-P.

43. Obiweluozor FO, Emechebe GA, Kim DW, Cho HJ, Park CH, Kim CS, Jeong IS. Considerations in the development of small-diameter vascular graft as an alternative for bypass and reconstructive surgeries: a review. Cardiovasc Eng Technol. 2020. https://doi.org/10.1007/s13239-020-00482-y.

44. Graham LM, Burkel WE, Ford JW, Vinter DW, Kahn RH, Stanley JC. Immediate seeding of enzymatically derived endothelium in dacron vascular grafts: early experimental studies with autologous canine cells. Arch Surg. 
1980;115:1289-94. https://doi.org/10.1001/archsurg.1980.0138011003 3005.

45. Boccafoschi F, Habermehl J, Vesentini S, Mantovani D. Biological performances of collagen-based scaffolds for vascular tissue engineering. Biomaterials. 2005;26:7410-7. https://doi.org/10.1016/j.biomaterials.2005. 05.052.

46. Patel A, Fine B, Sandig M, Mequanint K. Elastin biosynthesis: the missing link in tissue-engineered blood vessels. Cardiovasc Res. 2006;71:40-9. https://doi.org/10.1016/j.cardiores.2006.02.021.

47. Berglund JD, Nerem RM, Sambanis A. Incorporation of intact elastin scaffolds in tissue-engineered collagen-based vascular grafts. Tissue Eng. 2004;10:1526-35. https://doi.org/10.1089/ten.2004.10.1526.

48. Stegemann JP, Kaszuba SN, Rowe SL. Review: advances in vascular tissue engineering using protein-based biomaterials. Tissue Eng. 2007;13:260113. https://doi.org/10.1089/ten.2007.0196.

\section{Publisher's Note}

Springer Nature remains neutral with regard to jurisdictional claims in published maps and institutional affiliations.

- fast, convenient online submission

- thorough peer review by experienced researchers in your field

- rapid publication on acceptance

- support for research data, including large and complex data types

- gold Open Access which fosters wider collaboration and increased citations

- maximum visibility for your research: over $100 \mathrm{M}$ website views per year

At BMC, research is always in progress.

Learn more biomedcentral.com/submissions 